Jesús Moran Cepedano*

ORCID: 0000-0003-1939-8656

Rocca di Papa, Italia

\title{
La prospettiva antropologica di Chiara Lubich per il terzo millennio
}

\section{Chiara Lubich's Anthropological Perspective for the Third Millennium}

Summary: Today, we are witnessing the wide-ranging effects of a true anthropological emergency which, at all levels, calls for a new vision of the future in order to be addressed effectively and to change from being a huge challenge to becoming a new opportunity regarding the fate of future generations. To respond adequately to this task, it is necessary on the one hand to listen to authoritative voices that can interpret our times and, on the other, to refer to some essential phases of human history that came before the present phase. In an undertaking of this kind, it is of fundamental importance to reflect on how all this research has been received and understood within the ecclesial context and what consequences it has brought to the actual self-awareness of the Church. It is in this context that the anthropological and educative approach arising from the charism of Chiara Lubich and the experience of the Focolare Movement come into play.

* Jesús Moran Cepedano, filosofo e teologo. Dottorato in Teologia alla Pontificia Università Lateranense. Docente e consulente nel Dipartimento di Pastorale Universitaria della Pontificia Università Cattolica del Cile (1987-1991). Copresidente del Movimento dei Focolari (dal 2014). Contatto: Via di Frascati, 306. 00040 Rocca di Papa RM, Italia; e-mail: jesus. moran@focolare.org. 
Keywords: second modernity; anthropological emergency; the humanism of unity.

Sommario: Si assiste oggi ad effetti su larga scala di una vera emergenza antropologica che richiede a tutti i livelli una nuova visione prospettica per essere efficacemente affrontata e per trasformarsi da una grande sfida in nuova opportunità per le sorti delle future generazioni. Per poter essere all'altezza di tale compito, occorre da un lato ascoltare quelle voci autorevoli che interpretano il nostro tempo e dall'altro rifarsi ad alcune fasi essenziali della storia umana che hanno preceduto la fase attuale. In tale impresa è fondamentale riflettere su come tutto ciò sia stato recepito e interpretato all'interno del contesto ecclesiale e quali conseguenze abbia rappresentato per la stessa autocoscienza della Chiesa. E proprio in tale contesto si inserisce propriamente la proposta antropologico-educativa che emerge dal carisma di Chiara Lubich e dall'esperienza del Movimento dei Focolari.

Parole chiavi: seconda modernità; emergenza antropologica; umanesimo dell'unità.

Pienamente inoltrati nel terzo millennio della nostra era, assistiamo, oggi più che mai, ai primi effetti su larga scala di quella vera emergenza antropologica, preannunciata da alcuni decenni e che, ormai, investe tutti gli ambiti del sapere e dell'agire dell'essere umano. Di fatto, sembra che non si possa attualmente affrontare più alcuna problematica umana con garanzie minime di lucidità e coerenza, se non si ha il coraggio di andare a scavare prima nel sostrato antropologico alla base di essa.

Si tratta pertanto di acquisire questa visione prospettica che sola può garantire una dimensione che sia contemporaneamente profonda e sintetica. E ciò si presenta, allo stesso tempo, come una grande opportunità e una enorme sfida da cui possono dipendere, in gran parte, le sorti delle future generazioni. In tale ambito, come si inserisce la proposta antropologico-educativa che emerge dal carisma di Chiara Lubich e dall'esperienza del Movimento dei Focolari?

Occorre, evidentemente, partire dalla constatazione che la pedagogia si basa sempre su una antropologia, cioè su una visione e su un discorso sull'uomo (Aristotele), essendo proprio la scienza che studia l'uomo in maniera sistematica dal punto di vista dei suoi vari comportamenti all'interno di una società. E, a maggior ragione, dato che il discorso sull'uomo non è uni- 
voco, qualsiasi progetto educativo risente delle diverse correnti di pensiero in campo pedagogico, come risulta dalla storia stessa della pedagogia, sia per quanto riguarda la pratica educativa che la riflessione stessa sull'educazione.

Pertanto, conviene innanzitutto soffermarsi brevemente sul contesto culturale odierno con le sue contraddizioni e i suoi risvolti antropologici, così come viene interpretato da alcune voci autorevoli, per passare poi, a volo d'uccello, ad un breve excursus sulle grandi tappe della storia umana che hanno preceduto la fase attuale e che possono gettare luce su di essa.

In seguito, sarà necessario riflettere su come tutto ciò sia stato recepito e interpretato all'interno del contesto ecclesiale e quali conseguenze abbia rappresentato per la stessa autocoscienza della Chiesa per poter, infine, tentare di inquadrare sinteticamente la prospettiva antropologica che scaturisce dalla visione di Chiara Lubich ed evidenziare il contributo educativo che potrebbe derivare dalla prassi di vita che, da molti decenni, si sperimenta nel Movimento dei Focolari, lasciando agli studi accademici in corso il compito di sviscerarne i contenuti e i metodi.

\section{Le aporie della seconda modernità**}

Il processo che da oltre tre secoli si sta svolgendo in Occidente, al di là di tutte le sue varianti, conflittualità e contraddizioni, viene solitamente qualificato come processo di modernizzazione, che dal punto di vista antropologico può essere letto, in estrema sintesi, come un processo di individualizzazione.

Zygmunt Bauman, nel suo saggio dal significativo titolo Individualmente insieme, lo descrive in questi termini:

L'individualizzazione consiste nella trasformazione dell'identità umana da un qualcosa di 'dato' a un 'compito', e nell'attribuzione agli attori della responsabilità della realizzazione di questo compito e delle conseguenze (gli effetti collaterali) delle loro azioni. In altre parole consiste nell'istituzione di un'autonomia de iure (benché non necessariamente di un'autonomia de facto). Gli esseri umani non vengono più al mondo con delle identità definite ${ }^{1}$.

** Questo testo è una rielaborazione ampiamente riveduta e corretta di un precedente intervento al Convegno "Come in cielo, così in terra", svoltosi alla Pontificia Università Urbaniana di Roma, il 20 marzo 2010.

${ }^{1}$ Zygmunt Bauman, Individualmente insieme (Reggio Emilia: Diabasis, 2008), 31. 
A suo avviso, l'epoca moderna non consisterebbe in altro che in una sorta di corsa verso l'autodeterminazione. I risultati, però, non sembrano quelli che ci si poteva aspettare: "la capacità auto-affermativa delle donne e degli uomini individualizzati - prosegue - di regola, non raggiunge ciò che sarebbe richiesto da un'effettiva auto costituzione"'. E potremmo aggiungere che non stia, davvero, portando alla costituzione di una società più degna dell'uomo e più vivibile per tutti, tanto meno ad una convivenza tra uguali solidali.

Sembra, al contrario, che tale processo stia conducendo in generale ad una sorta di "disintegrazione della cittadinanza" 3 con conseguenze deleterie per gli stessi individui. Infatti ciò che alla fine ci troviamo davanti sono delle:

'comunità' fragili e di breve durata come pure emozioni disperse e vagolanti, che si spostano erraticamente da un obiettivo all'altro e che vagano nella vana ricerca di un approdo sicuro: comunità fondate su preoccupazioni condivise, su paure e odi comuni, ma in ogni caso comunità 'a tempo'4.

Facendo riferimento ad Ulrich Beck, sottolinea che ciò che sta emergendo da tale dissoluzione è:

l'io nudo, aggressivo, spaventato, alla disperata ricerca di aiuto e amore. In questa ricerca di se stesso e di una socialità solidale esso facilmente si perde nella giungla del sé. [...] Un essere allo sbando nella nebbia del suo sé è ormai incapace di prendere atto che questo isolamento, questo 'confinamento solitario dell'io' è una condanna di massa ${ }^{5}$.

E conclude:

Questo enorme divario tra il diritto all'autoaffermazione e la capacità di controllare i contesti sociali che rendono tale autoaffermazione possibile o irrealistica pare essere la principale contraddizione della 'seconda modernità', un'epoca che, attraverso tentativi ed errori, riflessioni critiche e audaci sperimentazioni dovremo imparare collettivamente ad affrontare collettivamente ${ }^{6}$.

2 Ibidem, 35.

3 Ibidem, 37.

4 Ibidem, 38.

5 Ibidem, 39.

${ }^{6}$ Ibidem. Altri autori parlano di neoindividualismo per riferirsi al contesto sociale in cui vivono gli uomini e le donne del nostro tempo. Questo neoindividualismo sarebbe il prodotto 
A Bauman, che si fa portavoce dell'idea di "radicalizzare la modernità" come via d'uscita, fa eco Jeremy Rifkin, qualche anno dopo, nel suo libro La civiltà dell'empatia, nel quale offre una lettura della storia delle civiltà vista dall'angolatura di questo valore e azzarda un approfondimento delle qualità umane che potrebbero farci uscire dalla crisi attuale.

Civiltà sempre più complesse e affamate di risorse - afferma - permettono all'uomo di comprimere lo spazio e il tempo, di espandere [...] una sorta di sistema nervoso centrale collettivo per abbracciare aree più vaste dell'esistenza. Facciamo tutto questo per trovare significato attraverso l'appartenenza a un ambito più ricco e profondo della realtà ${ }^{8}$.

E avverte:

Un'idea radicalmente nuova di natura umana sta lentamente emergendo e acquistando forza, con implicazioni rivoluzionarie sul modo in cui, nei secoli a venire, interpreteremo e organizzeremo le nostre relazioni sociali e ambientali. Abbiamo scoperto l'Homo empaticus'.

Ad ulteriore dimostrazione di quanto impellente sia la questione, non si può non accennare almeno ai suoi risvolti nel campo dell'educazione che,

paradossale di una società sempre più globalizzata e quindi iper-relazionata. Questi i suoi tratti principali: la disintegrazione e la mancanza di coesione sociale; il sorgere di una nuova classe di parias della civiltà globale; la conversione della capacità economica del consumare come criterio fondamentale di inclusione o esclusione sociale; la privatizzazione dei vincoli sociali; la disuguaglianza come fattore determinante di esclusione sociale; l'emergenza di un'etica dell'arricchimento privato al di sopra di tutto; l'apparire di un individualismo senza mala coscienza che sbocca in un nichilismo distruttivo e nel paradosso della privazione e del non possedere; la distruzione della dimensione collettiva, solidale e democratica dei rapporti sociali; il trionfo del privato sul collettivo; la configurazione di una specie di autismo sociale fra gli esseri umani; la tendenza alla radicalizzazione culturale, etnica, comunitaria, urbana, religiosa o, in generale, identitaria dei gruppi socialmente vulnerabili e per questo facilmente manipolabili [Cf. Maria José Fariñas Dulce, "Neoindividualismo y desigualdad", El País, mercoledì, 5 gennaio 2005, https://elpais.com/diario/2005/01/05/opinion/1104879608_850215. html; 20.09.2018].

${ }^{7}$ Cf. Bauman, Individualmente, 39.

8 Jeremy Rifkin, La civiltà dell'empatia (Milano: Mondadori, 2010), 39.

9 Ibidem, 42. 
secondo Martha Nussbaum, si trova nel mezzo di una crisi di proporzioni senza precedenti e di natura globale:

Mi riferisco ad una crisi che passa inosservata, che lavora in silenzio, come un cancro; una crisi destinata ad essere, in prospettiva, ben più dannosa per il futuro della democrazia: la crisi mondiale dell'istruzione. Sono in corso radicali cambiamenti riguardo a ciò che le società democratiche insegnano ai loro giovani, e su tali cambiamenti non si riflette abbastanza. Le nazioni sono sempre più attratte dall'idea del profitto; esse e i loro sistemi scolastici stanno accantonando, in maniera del tutto scriteriata, quei saperi che sono indispensabili a mantenere viva la democrazia ${ }^{10}$.

L'analisi esplicitamente critica del panorama mondiale che la Nussbaum porta avanti nel suo testo la conduce ad una conclusione - solo apparentemente - sorprendente che lei mutua da Tagore quando - mettendo in guardia dalla frenetica corsa al possesso delle cose materiali - dice che, così, ci si avvia ad un processo: "di lento suicidio dovuto al disseccamento dell'anima"11. Per quanto possa sembrare strano o, almeno, curioso il riferimento ad un termine di natura squisitamente religiosa, la filosofa americana precisa in qual senso tale termine può essere, comunque, compreso da tutti e accettabile.

Esso vuole rappresentare:

le capacità di pensiero e immaginazione che ci rendono umani, e che fanno delle nostre relazioni qualcosa di umanamente ricco, non relazioni di semplice uso e manipolazione. [...] La capacità di pensare criticamente, la capacità di trascendere i localismi e di affrontare i problemi mondiali come 'cittadini del mondo'; e, infine, la capacità di raffigurarsi simpateticamente la categoria dell'altro ${ }^{12}$.

In sostanza, si tratta di tener conto che ogni relazione avviene sempre all'interno di uno spazio antropologico e che necessita del dovuto rispetto e della imprescindibile cura. Come non pensare a don Lorenzo Milani e al cartello che aveva appeso sulla parete della sua scuola di Barbiana con su scritto "I care"?

\footnotetext{
${ }^{10}$ Martha C. Nussbaum, Non per profitto (Bologna: Il Mulino, 2011), 21.

11 Ibidem.

12 Ibidem, 25-26.
} 
E per concludere questa breve ricognizione circa il contesto in cui ci si trova, oggi, a doversi confrontare - anche e soprattutto in campo educativo riemergono illuminanti queste poche e significative parole di George Steiner sulla funzione dei maestri:

ho sentito crescere in me l'incertezza riguardo alla legittimità, alle verità sottostanti, di questa 'professione'. Metto questa parola tra virgolette per segnalarne le radici complesse. Che affondano in antecedenti religiosi e ideologici. Il mestiere del 'professore', un termine in qualche misura di per sé opaco, abbraccia ogni sfumatura possibile tra gli estremi di una vita di routine, disincantata e un esaltato senso di vocazione ${ }^{13}$.

\section{Le tappe fondamentali dello sviluppo antropologico in Occidente}

Se la lettura di fondo di questa fase dell'umanità appare essere sostanzialmente accertata - al di là delle evidenti differenze legate ai diversi ambiti culturali - ciò che è, tuttavia, di difficile interpretazione è l'eziologia profonda dei fenomeni e soprattutto la ricerca di possibili vie di soluzione che sono ancora in piena fase di elaborazione, non senza rischi di derive di varia natura. Per fare un passo avanti, occorre provare a contestualizzare questa seconda modernità con i suoi fenomeni di individualizzazione e nella quale si affaccia - se si accetta la tesi di Rifkin - un nuovo tipo d'uomo, all'interno del grande percorso di sviluppo dell'autocoscienza dell'umanità in cui, fra le tante tappe, si possono certamente evidenziare alcune fasi decisive. Una caratterizzazione efficace e sintetica di questo percorso - soprattutto per quel che riguarda l'Occidente - la si trova in molti scritti di Giuseppe M. Zanghí ${ }^{14}$ che per decenni ne ha fatto il centro della sua riflessione. Per lui, fondamentalmente, tutte le diverse concezioni dell'uomo che la storia occidentale ha elaborato finora, possono essere ricondotte a due grandi visioni antropologiche legate al rispettivo modo di intendere e vivere il rapporto con l'Assoluto e quindi il corrispondente esercizio del pensare e dell'agire. Naturalmente

${ }_{13}$ George Steiner, La lezione dei maestri (Milano: Garzanti, 2004), 9.

${ }^{14}$ Cf. Giuseppe Maria Zanghí, "Il pensare come amore. Verso un nuovo paradigma culturale", Nuova Umanità 145 (2003): 1-19; Giuseppe Maria Zanghí, Notte della cultura europea (Roma: Città Nuova, 2007); Giuseppe Maria Zanghí, Leggendo un carisma. Chiara Lubich e la cultura (Roma: Città Nuova, 2015). 
non è possibile separare esplicitamente tali concezioni perché non sono compartimenti stagno, esattamente definiti nel tempo e delimitati nello spazio, ma solo delle grandi linee storiche (siamo più nella filosofia della storia che nella storia stessa) che, in genere, convivono autonomamente pur continuando ad intersecarsi le une con le altre in fasi successive.

La prima fase è quella caratterizzata dal "pensare come mito" 15 , tipica del primo lungo stadio di sviluppo dell'umanità. Nella cultura mitologica, di tutte le tradizioni culturali, il soggetto pensante è il gruppo e l'oggetto pensato è il divino, entrambi - pensante e pensato - sempre immersi e costantemente avvolti dalla luce di quest'ultimo. La categoria fondamentale del pensiero è, dunque, la memoria che riproduce la realtà originaria nella quale si vuole sempre restare. Primeggia il Bene ed emerge la figura della Grande Madre, che tutto custodisce e nutre.

Tratti fondamentali di tale concezione teorica ed esperienza esistenziale sono:

- l'immersione nel divino, concepito come grembo dal quale l'uomo non si distingue;

- la comunione profonda con tutto l'universo;

- il totale senso di dipendenza dell'uomo dall'Assoluto;

- la sacralizzazione e la ritualizzazione dell'esistenza;

- il ruolo dominante della classe sacerdotale;

- la percezione diffusa di una lacerazione misteriosa nell'Uno quando si deve tener conto delle singolarità.

La seconda tappa è quella che emerge con il passaggio al "pensare come logos" ${ }^{\text {"16 }}$. All'incirca verso il VI secolo a.C., inizia quella che Karl Jaspers ha chiamato epoca assiale, che vede il sorgere - quasi contemporaneo e davvero sorprendente - di fenomeni simili in tutte le tradizioni culturali: il profetismo in Israele, Buddha e le Upanishad in India, Confucio e Lao Tze in Cina, Zoroastro in Persia, i presocratici in Grecia.

Evidenti le differenze dei suoi caratteri essenziali rispetto alla fase precedente:

- l'uomo si pone di fronte al divino, percepito come distinto da sé;

- incomincia un percorso di indipendenza e autonomia;

- sorge il logos come modo di pensare il divino;

- il pensare diventa un esercizio discorsivo e non è più soltanto una azione di contemplazione o immersione nel divino;

${ }^{15}$ Cf. Zanghí, "Il pensare", 2-6.

16 Ibidem, 6-11. 
- emergono le figure dei saggi e dei pensatori;

- inizia ad eclissarsi il senso della caduta originaria e del bisogno di una salvezza.

Nella cultura del logos il soggetto pensante è l'uomo singolo e non più il gruppo. Si tratta di concepire il tutto ma a partire dal singolo e quindi dal logos che egli concepisce. L'oggetto pensato sarà la molteplice e varia e mutevole realtà delle cose. Il tutto - pensante e pensato - sempre più nella luce della ragione. E sarà questa cultura che dopo circa due millenni, almeno in Occidente, approda alla modernità che radicalizzerà il predominio della ragione fino al climax dell'idealismo hegeliano, seguito dalla reazione irrazionalista, poi dal nichilismo e infine dalla post-modernità.

Si potrebbe anche dire, usando altre ermeneutiche e sempre con la dovuta cautela - per evitare il rischio di una eccessiva ipersemplificazione - che la prima tappa definisce la dimensione prettamente cosmo-teocentrica tipica dello sviluppo della coscienza dell'umanità nel mondo antico mentre la seconda si inoltra in quella logo-antropocentrica che ha caratterizzato, fino ai nostri tempi, la modernità con tutte le sue enormi conquiste e che, ormai da alcuni decenni, ha lasciato il posto ad uno scenario completamente inedito.

È proprio questo lo scenario situato nell'orizzonte di quella seconda modernità cui abbiamo precedentemente accennato, soprattutto nell'interpretazione di Bauman e Rifkin. Il processo d'individualizzazione sottolineato da Bauman e l'emergere di quello che Rifkin chiama homo empaticus si collocano esattamente al crocevia della storia nello scorcio di questo terzo millennio e sembrano indicare che - pur tra innumerevoli difficoltà e possibili pericolose involuzioni - l'umanità è incamminata verso una terza tappa che vira decisamente nella direzione di quella che potremmo definire la dimensione personalistica.

A questo punto si pone una questione seria, perché - se si vuole cogliere la vera portata di ciò che sta accadendo - non si può eludere un problema tipicamente metafisico dal quale dipende ogni possibile considerazione sulla natura della convivenza umana. Come sottolineato da Xavier Zubiri: "il vero problema della convivenza sta nel fatto che ci si dica in che misura e in quale forma gli altri formano parte della mia realtà e, reciprocamente, in che misura io formo parte della realtà degli altri" ${ }^{17}$. Siamo, quindi, di fronte ad una questione di fondamenti.

17 Xavier Zubiri, Tres dimensiones del ser humano: individual, social, histórica, ed. Jordi Corominas (Madrid: Alianza Editorial - Fundación Xavier Zubiri, 2006), 51. Infatti, per il pensatore spagnolo: "gli uomini sono costitutivamente co-reali e costitutivamente non vuol 


\section{Alcune sfide per il cristianesimo e per la Chiesa}

Ora, come tutto ciò è stato recepito e interpretato all'interno del contesto ecclesiale e quali conseguenze ha comportato per la stessa autocoscienza della Chiesa e, quindi, per la sua azione?

Non si può non partire dalla constatazione che il cristianesimo è sorto proprio nel pieno della fioritura della fase culturale del logos, con la rivelazione del Verbo eterno che s'incarna per indicare la strada della vera divinizzazione percepita - secondo Zanghí - solo diffusamente nel mito e mai raggiunta, invece, dalla stessa cultura del logos a causa o dell'inevitabile ripiegamento della ragione su se stessa o della sua incapacità di superare la realtà del molteplice nell'Uno e della distinzione nell'unità.

Siamo nel cuore della riflessione che - sia pure in forme diverse e persino contrapposte - l'Occidente e l'Oriente hanno elaborato per quasi due millenni e che conduce ad una aporia definitiva: quella dell'inevitabile coesistenza tra la realtà dell'Essere e la dimensione del Non-Essere, che Zanghí esplicita sinteticamente con rara e puntuale lucidità:

L'uomo $[\ldots]$ ha compiuto questo percorso, iniziato nel mito e maturato nel logos, aggiungendovi di suo 'il peccato'. Quel NON misterioso, presentito già nel mito come intimo alla realtà del divino (e che il Logos incarnato rivelerà come l'Essere stesso di Dio, la Trinità, Amore-aperto-nel-Dono), è stato sentito dall'uomo 'tentato', ed è per lui diventato il non negativo del rigetto. Quasi che l'uomo non sia stato per sé capace di sostenere la forza dell'Amore che è Dio Trinità nel fuoco dell'unità-distinzione: attendeva il Logos eterno nella carne creata del Logos incarnato ${ }^{18}$.

È solo in questo orizzonte, infatti, che si può situare correttamente il percorso del passaggio dall'individuo alla persona che è tutt'altro che scontato e non può non fare i conti con il cristianesimo. Così come il cristianesimo, nel suo attuarsi in questo terzo millennio, non può non fare i conti con la seconda modernità, come affermato con forza e lungimiranza sor-

dire che lo siano perché non possono farne a meno, se non che ciò che ognuno è, è co-reale con gli altri per ragione della sua propria realtà. La convivenza umana è un carattere specifico della realtà propria della sostantività umana in quanto tale".

${ }^{18}$ Zanghí, "Il pensare", 10. 
prendente da Giovanni Paolo II, ancora nel 1982, al V Simposio dei Vescovi d'Europa:

Scopriamo forse non senza meraviglia che le crisi e le tentazioni dell'uomo europeo e dell'Europa sono crisi e tentazioni del cristianesimo e della Chiesa in Europa. [...] In questa luce il cristianesimo può scoprire nell'avventura dello spirito europeo le tentazioni, le infedeltà e i rischi che sono propri dell'uomo nel suo rapporto essenziale con Dio in Cristo ${ }^{19}$.

In queste parole profetiche - pronunciate in tempi non sospetti - troviamo una luminosa chiave di lettura di quello che è accaduto negli ultimi decenni e che ha spinto la Chiesa - "esperta in umanità" ${ }^{20}$ e ancorata alla sapienza del Vangelo - a interrogarsi sempre più circa le prerogative sul versante dei processi di umanizzazione contenute nel messaggio di cui è custode, in modo da accompagnare la vicenda degli uomini e delle donne nell'attuale complessa congiuntura storica. E non potrebbe essere diversamente proprio perché anche la Chiesa viene provata, nei suoi figli, dalla drammaticità dei processi storici, ed è costretta ad ammettere le sue ombre e le sue luci, le sue conquiste e le sue sconfitte, i suoi fallimenti e le sue speranze. In particolare l'emergenza antropologica cui oggi assistiamo - e che investe in pratica tutti gli ambiti dell'agire e dell'essere umano - rappresenta per essa una vera sfida. Non per nulla, il pontificato di Giovanni Paolo II si era aperto con l'enciclica programmatica Redemptor hominis, nella quale egli aveva ribadito con forza il fatto che l'uomo è e sarà sempre il cammino della Chiesa. Nell'enciclica riecheggiava il paragrafo 22 della Gaudium et spes: "Cristo, che è il nuovo Adamo, proprio rivelando il mistero del Padre e del suo amore svela anche pienamente 1'uomo a se stesso e gli manifesta la sua altissima vocazione" ${ }^{\text {"21. }}$. Il mistero dell'uomo, infatti, si cela nel mistero dell'uomo-Dio che lo rivela ${ }^{22}$. E si era concluso, fondamentalmente, con il grande giubileo del duemila e con la Lettera apostolica Novo Millennio Ineunte in cui - da una prospettiva che potremmo chiamare più intra-ecclesiale - Giovanni Pa-

19 Giovanni Paolo II, "V Simposio dei Vescovi d'Europa", L'Osservatore Romano, 7 ottobre 1982.

20 Paolo VI, Populorum progressio (Città del Vaticano: Libreria Editrice Vaticana, 1967), n. 13.

${ }^{21}$ Concilio Vaticano II, Gaudium et spes (Città del Vaticano: Libreria Editrice Vaticana, 1965), n. 22.

${ }^{22}$ Cf. Ibidem. 
olo II richiamava la Chiesa a quella dimensione profetica che, sola, può renderla lievito e sale nella massa della società del Terzo Millennio. E lo faceva indicando il passo ormai ineludibile e necessario di quella che egli chiama "ecclesiologia di comunione"23.

Questa, già preconizzata come dottrina dal Vaticano II - e cioè come autocoscienza della Chiesa - deve ora diventare prassi concreta e manifesta dei cristiani. E per essere più efficace, Giovanni Paolo II non teme di proporre la creazione di "scuole di ecclesiologia di comunione" 24 , dove si possa imparare e praticare la vita comunitaria nella sua vera essenza.

$\mathrm{Su}$ questa linea Benedetto XVI - consapevole, come pochi, del clima culturale di questi primi anni del terzo millennio - comincia il suo cammino a guida della Chiesa riportando all'attenzione, con forza, il novum più dirompente del cristianesimo: Deus caritas est, rivelando, così, il volto vero di Dio e il suo stare davanti $a$ e nelle sue creature. Amore che si fa speranza per l'uomo (Spe salvi), e cioè, pegno di giustizia terrena e gloria eterna. Amore che s'identifica con la verità ${ }^{25}$ e che ad essa approda perché da essa proviene. Ed è particolarmente nella Caritas in veritate che troviamo più sviluppata questa sottolineatura antropologica. Al n. 75 si dice con singolare pregnanza: "oggi occorre affermare che la questione sociale è diventata radicalmente questione antropologica" 26 . Se "il mondo soffre per mancanza di pensiero afferma Benedetto XVI, riprendendo il motto di Paolo VI nella Populorum progressio - questo vuoto può essere colmato solo con una nuova sintesi umanistica"27, con un reale rinnovamento che superi l'appiattimento che pervade la cultura odierna. Si tratta di puntare ad uno sviluppo morale, che necessita anche di uno sforzo d'intelligenza e di amore, riuniti in una nuova sintesi, per "fare interagire i diversi livelli del sapere umano" 28 in una "interdisciplinarietà ordinata" 29 . Urge, allora, un allargamento ${ }^{30}$ del concetto di

${ }^{23}$ Giovanni Paolo II, Novo Millennio Ineunte (Città del Vaticano: Libreria Editrice Vaticana, 2001), n. 43.

${ }^{24}$ Giovanni Paolo II al Sinodo Romano, citato in: Piero Coda, "I movimenti ecclesiali, dono dello Spirito", in: Pontificium Consilium pro Laicis, I movimenti nella Chiesa (Città del Vaticano: Libreria Editrice Vaticana, 1999), 96.

${ }^{25}$ Benedetto XVI, Caritas in Veritate (Città del Vaticano: Libreria Editrice Vaticana, 2009).

26 Ibidem, n. 75.

27 Ibidem, n. 21.

28 Ibidem, n. 30.

${ }^{29}$ Ibidem.

${ }^{30}$ Cf. Ibidem, n. 31. 
ragione per renderla capace di accogliere la vera realtà dell'uomo e le sfide a cui le nuove dinamiche mondiali lo stanno sottoponendo ${ }^{31}$.

Con uno sguardo sapienziale sull'intero processo, Benedetto XVI sostiene che:

La transizione insita nel processo di globalizzazione presenta grandi difficoltà e pericoli, che potranno essere superati solo se si saprà prendere coscienza di quell'anima antropologica ed etica, che dal profondo sospinge la globalizzazione stessa verso traguardi d'umanizzazione solidale. [...] Ciò consentirà di vivere ed orientare la globalizzazione dell'umanità in termini di relazionalità, di comunione e di condivisione ${ }^{32}$.

Ed è proprio in questo panorama mondiale - ulteriormente compromesso dalla crisi economica scoppiata nel 2008 - che si situa coerentemente il pontificato di papa Francesco, in tempi in cui l'emergenza antropologica non ha fatto che riacutizzarsi e ingigantirsi. Già nella prima enciclica Lumen fidei, egli sottolinea la particolare relazione tra verità e amore che consiste nell'essere l'una nell'altro:

Chi ama capisce che l'amore è esperienza di verità, che esso apre i nostri occhi per vedere tutta la realtà in modo nuovo, in unione con la persona amata $[\ldots]$ Si tratta di un modo relazionale di guardare il mondo, che diventa conoscenza condivisa, visione nella visione dell'altro e visione comune su tutte le $\operatorname{cose}^{33}$.

In seguito, sulla base della precedente e ricca tradizione magisteriale, nell'Esortazione apostolica Evangelii gaudium - vero manifesto del suo pontificato, come stiamo verificando col passare del tempo - egli, tra moltissimi altri spunti, si sofferma sulla necessità di "nuove relazioni generate da Cristo" e di "mistica del vivere insieme":

Oggi, quando le reti e gli strumenti della comunicazione umana hanno raggiunto sviluppi inauditi, sentiamo la sfida di scoprire e trasmettere la "mistica" del vivere insieme, di mescolarsi, di incontrarci, di prenderci in braccio, di appoggiarci, di partecipare a questa marea un po' caotica che può trasformarsi

\footnotetext{
${ }^{31}$ Cf. Ibidem, n. 33.

32 Ibidem, n. 42.

${ }^{33}$ Francesco, Lumen Fidei (Città del Vaticano: Libreria Editrice Vaticana, 2013), n. 27.
} 
in una vera esperienza di fraternità, in una carovana solidale, in un santo pellegrinaggio ${ }^{34}$.

Come sottolineato, rispettivamente, nei saggi di Peter Hünermann e Piero Coda, all'interno della collana La teologia di papa Francesco, da un lato, possiamo verificare come egli si muova esplicitamente a partire dal cuore della moderna antropologia filosofica e dalla sua determinazione fondamentale sull' "essere-nel-mondo dell'uomo" ${ }^{35}$; dall'altro, come la sua teologia

sia ecclesiale e insieme esistenziale, spirituale e insieme kerigmatica, mistica e insieme sociale. Una sintesi originale e personalissima, che sprigiona una grande attrattiva ed incisività. Come mostrano la gioia e l'efficacia che irradia ${ }^{36}$.

Tale approccio caratteristico della sua personale esperienza e del contesto culturale da cui proviene, permea tutto il suo orizzonte antropologico così esplicitamente connotato dalla dimensione pastorale che egli manifesta in tutto ciò che dice e che opera. Tuttavia per essere colto in maniera organica va riferito ad una precisa idea circa la natura della realtà creaturale come afferma, per esempio, in questo sintetico passaggio della Laudato si': "ogni creatura porta in sé una struttura propriamente trinitaria" ${ }^{37}$.

Infine, nella recentissima Costituzione apostolica Veritatis gaudium, circa le università e le facoltà ecclesiastiche, tutto ciò trova uno sbocco anche sul fronte più propriamente culturale e pedagogico. Egli parla della necessità di una "rivoluzione culturale" 38 nel segno di una dilatazione della ragione ${ }^{39}$, nella quale le università e le facoltà ecclesiastiche sono chiamate a giocare un ruolo di primo piano. E indica - come risposta alla crisi antropologica e socio-ambientale - la necessità di "cambiare il modello di sviluppo globale" e "ridefinire il progresso" 40 all'insegna di quattro criteri di fondo:

${ }^{34}$ Francesco, Evangelii gaudium (Città del Vaticano: Libreria Editrice Vaticana, 2013), n. 83 .

${ }^{35}$ Cf. Peter Hünermann, Uomini secondo Cristo oggi. L'antropologia di papa Francesco (Città del Vaticano: Libreria Editrice Vaticana, 2017), 40.

${ }^{36}$ Piero Coda, La Chiesa è il Vangelo. Alle sorgenti della teologia di papa Francesco (Città del Vaticano: Libreria Editrice Vaticana, 2017), 11.

37 Francesco, Laudato sii (Città del Vaticano: Libreria Editrice Vaticana, 2015), n. 239.

${ }^{38}$ Cf. Francesco, Veritatis gaudium (Città del Vaticano: Libreria Editrice Vaticana, 2018), n. 3.

${ }^{39}$ Cf. Ibidem, n. 2.

${ }^{40}$ Ibidem. 
a) la "contemplazione e [...] introduzione spirituale, intellettuale ed esistenziale nel cuore del kerigma"; b) il "dialogo a tutto campo"; c) "l'inter - e la trans-disciplinarietà"; d) il "fare rete tra le diverse istituzioni" 4 .

\section{Un umanesimo per il terzo millennio}

Il quadro fin qui delineato, sia pur sommariamente, pone una questione di fondo per non restare unicamente sul piano dei buoni propositi: quella di disporre di una articolata e consistente formulazione di cosa sia la relazione interpersonale e di come si debbano rifondare e ridefinire i rapporti all'interno delle comunità di ogni genere. Ciò è oltremodo urgente sul piano educativo e pedagogico, come molti segnali di allarme nel mondo giovanile sembrano indicare. E se questa esigenza è avvertita a tutti i livelli, all'interno della compagine ecclesiale - come abbiamo visto - ha sollecitato interventi ripetuti del magistero degli ultimi pontefici che ne hanno fatto una priorità assoluta. Allo stesso tempo, volendo cogliere i "segni dei tempi" 42 , non si può non sottolineare quanto l'azione dello Spirito abbia contribuito allo sforzo messo in opera dalla Chiesa riconoscendo carismi adatti ai tempi, facendo sorgere realtà che li incarnassero nella nostra storia.

Questi doni, venendo dall'alto, oltre a costituire in se stessi delle risposte ai grandi problemi umani d'ogni tempo, si situano in rapporto dinamico con il Magistero e con la vita della Chiesa tutta, non mediante una sinergia solo affettiva o strategica, quanto strettamente teologica, poiché rendono manifesta la co-essenzialità tra doni carismatici e doni ministeriali nella vita della Chiesa ${ }^{43}$. È, pertanto, in questo quadro interpretativo che possiamo cogliere il carisma dell'unità donato da Dio - tramite Chiara Lubich - alla Chiesa e all'umanità, proprio nel periodo di avvicinamento alla transizione al Terzo Millennio. Un carisma che, per il suo dinamismo interno, sembra possedere anche una vera riproposizione antropologica in piena consonanza con le esigenze e le sfide del nostro tempo.

Secondo la nota affermazione di Hans Urs von Balthasar, per il quale ogni grande carisma è uno sguardo onnicomprensivo sulla realtà a par-

\footnotetext{
${ }^{41}$ Ibidem, n. 4.

${ }^{42}$ Concilio Vaticano II, Gaudium, n. 4.

${ }^{43}$ Cf. Congregazione per la dottrina della fede, Iuvenescit Ecclesia (Città del Vaticano, 16 maggio 2016), nn. 10 e 13.
} 
tire dal centro della Rivelazione ${ }^{44}$, a buon diritto si può cogliere nel carisma di Chiara Lubich anche una ulteriore versione, adatta ai nostri giorni, di quell'umanesimo cristiano che ha caratterizzato sempre, in ogni epoca, la grande Tradizione della Chiesa stessa: l'umanesimo dell'unità. E, all'interno di esso, individuare le possibili indicazioni che ne emergono sul fronte più strettamente pedagogico ed educativo ${ }^{45}$. Ovviamente tale obiettivo non è lo scopo di questo articolo, anche perché già da qualche anno sono cominciati studi a livello accademico in tale direzione, a cui occorrerà riferirsi per evidenziare la specifica portata pedagogica del carisma e della sua incarnazione nella prassi di vita del Movimento dei Focolari e di tutte le varie realtà che ad esso si ispirano e che non possono non partire direttamente dal ripercorrere, intanto, la sua vicenda storica, così intrecciata con la dimensione prettamente pedagogica, avendo Lubich, da giovane, fatto per vari anni la maestra.

In queste pagine si è voluto solamente inquadrare tale prospettiva e la sua portata nell' orizzonte culturale ed ecclesiale odierno, suggerendo alcune piste di riflessione, per poter attingere a tutta la ricchezza di teoria e prassi che ne potrebbe emergere. In questo senso, e in linea di massima, occorrerebbe partire dall'educazione di Chiara Lubich nell'infanzia e nell'adolescenza (Trento, 1920-1936), dalla sua formazione culturale e cristiana, compresa la formazione professionale alle magistrali (Trento, seconda metà degli anni 1930), e da Lubich maestra elementare e maestra delle aspiranti dell'Azione Cattolica prima e del Terz'Ordine Francescano poi (1938-1943) ${ }^{46}$.

Evidentemente è con la svolta avvenuta in lei, nel contesto della seconda guerra mondiale, e con l'adesione di tante persone al suo stile di vita e ai suoi insegnamenti - e, quindi, con la nascita del Movimento dei Focolari che inizia quel percorso umano e spirituale che, nel tempo, si arricchirà di intuizioni, esperienze, realizzazioni, e che farà emergere, tra il resto, quello che sempre più sembra costituire un vero e proprio progetto educativo inculturato nei più vari contesti geografici, sociali e religiosi. E poiché - come

${ }^{44}$ Cf. Hans Urs von Balthasar, Teologica, vol. III (Milano: Jaca Book, 1992), 22.

${ }^{45}$ Chiara Lubich, Educazione come vita, ed. A. Vincenzo Zani (Brescia: La Scuola, 2010), 42-48; cf. Francesco Chatel, “Alla scuola di chi?”, Nuova Umanità 6/180 (2008): 613-621; Pino Quartana, "Introduzione. Avere il Maestro tra di noi”, in: Chiara Lubich, Una famiglia per rinnovare la società (Roma: Città Nuova, 1993), 105-108; Donato Falmi, "Presentazione della collana Studi e Documenti", in: Qui c'è il dito di Dio. Carlo de Ferrari e Chiara Lubich: il discernimento di un carisma, ed. Lucia Abignente (Roma: Centro Chiara Lubich - Città Nuova, 2017), 5-8.

${ }^{46}$ Cf. Nino Carella, Silvia prima di Chiara. La ricerca di una strada nuova (Roma: Città Nuova, 2014). 
già sottolineato ampiamente - ogni proposta pedagogica si fonda su una antropologia, essenziale diventa cogliere e sviscerare le linee portanti di tale antropologia, che non può non essere cristiana nel suo senso più pieno e più aperto, intendendo l'aggettivo cristiana non con una accezione alternativa, isolante o, peggio, elitaria ed escludente - nei confronti del mondo dell'educazione - ma come quella prospettiva che radica all'interno e in dialogo con esso. Si tratta, pertanto, prima di tutto, di far emergere la portata culturale del carisma di Chiara Lubich e non relegarlo solamente nell'ambito, pur importante e primario, della spiritualità. In tale prospettiva chi - per primo e per tutta la vita - ha operato un tale tentativo con organicità e competenza - oltre che partecipazione diretta, essendo stato uno dei primi compagni di Lubich è stato Giuseppe M. Zanghí, che abbiamo già citato in precedenza nel breve excursus storico sulle vicende della cultura occidentale. Ed è ancora a lui che facciamo riferimento per cogliere quello che riteneva essere la vera novità del carisma e della persona di Chiara Lubich. Infatti, l'esito irrimediabilmente nichilista di buona parte della cultura odierna è legato, secondo lui, ad una incompleta e per certi versi carente lettura dell' evento cristiano e, in particolar modo, dello "scandalo della croce" con il misterioso e inquietante grido di abbandono di Gesù prima di morire (Cf. Mt 27, 46; Mc 15, 34; Sal 22, 2).

Per Zanghí, l'unica possibilità di resurrezione della cultura odierna è nella sfida che tale realtà pone, non solo alla ragione postmoderna autosufficiente e autoreferenziale, ma soprattutto alla stessa fede dei credenti. L'unica possibilità di rovesciamento radicale del nichilismo odierno consiste nella possibilità di rovesciare il NON negativo della cultura del logos nel NON positivo della cultura dell' Amore che egli considera essere la grande rivelazione che lo Spirito ha fatto a Chiara Lubich svelandole il mistero dell'abbandono di Gesù in croce. É la terza tappa fondamentale dello sviluppo del pensiero dell'umanità che lui chiama: "il pensare come amore"47. Sarebbe quella "cultura dello Spirito" 48 , che ci introduce nel mistero della Trinità,

${ }^{47}$ Cf. Zanghí, "Il pensare”, 11-19.

${ }^{48}$ Non si tratta, evidentemente, di nulla di simile alla teologia della storia di Gioacchino da Fiore con il suo taglio spiritualistico ed escludente. Quando Zanghí parla dello Spirito si riferisce a quello Spirito del Figlio che grida: "Padre" (Cf. Gal 4, 6). Siamo sempre all'interno di una prospettiva di progressiva penetrazione nella verità una del mistero di Dio, la quale, sebbene ci venga donata tutta intera sin dall'inizio, necessita di una lenta assimilazione da parte dell'uomo. E questo come dono di Dio stesso, giacché è sempre Lui a condurre tale processo. Benedetto XVI lo ha espresso molto bene, nell'Udienza Generale del 10 marzo 2010, citando San Bonaventura: "Opera Christi non deficiunt, sed proficiunt" (Le opere di Cristo non vanno indietro, non vengono meno, ma progrediscono). 
grembo d'infinito Amore che racchiude e riflette il vero volto di Dio e il vero volto dell'uomo. Solo abitando questo luogo è possibile cogliere la dinamica dei rapporti trinitari ad intra per riconoscerli, poi, ad extra nella creazione e nella storia dell'umanità che, con l'incarnazione del Verbo, ha subito una vera e propria transizione di fase.

In un interessante testo presente nel suo volume d'inediti postumi sul problema teologale dell'uomo, il filosofo spagnolo Xavier Zubiri - parlando del rapporto fra il cristianesimo e l'uomo attuale - contesta la visione tradizionale secondo cui il cristianesimo sarebbe primariamente e formalmente una religione della salvezza, perché ciò equivarrebbe a porre l'accento quasi esclusivamente sulla costitutiva situazione d'indigenza dell'uomo. Invece, a suo avviso, il cristianesimo sarebbe in modo primario e formale - e cita Rm 2, 20 - una morphosis, "una conformazione divina dell'uomo intero [...] una deiformità" "49. Questo vuol dire che se il cristianesimo raggiunge l'uomo nella sua condizione d'indigenza, è per aprire e indirizzare questa limitatezza verso la sua vera chiamata alla divinizzazione. Nel nostro tempo in cui come diceva Bauman - nessun uomo viene al mondo con un'identità definita e in cui l'autodeterminazione è avvertita come valore fondamentale, questa affermazione del pensatore basco, penso, vada presa sul serio per la sua lungimiranza e, soprattutto, per la sfida che pone alla teologia. Infatti, così egli conclude le sue riflessioni sull'oggi: "Il punto di coincidenza fra l'uomo attuale e il cristianesimo non è l'indigenza della vita ma la sua pienezza" ${ }^{\prime \prime}$.

\section{La prospettiva di Chiara Lubich}

In tale prospettiva emerge evidente come il carisma dell'unità sia un particolare dono dello Spirito proprio per questi tempi di pienezza nell'accezione del filosofo basco, per il quale ciò sta a significare una nuova autocoscienza dell'uomo nonostante tutte le difficoltà e limitazioni. L'uomo d'oggi, infatti, sembra voler attingere al massimo delle sue potenzialità liberato da ogni infantile dipendenza da ciò che gli è esteriore, ma allo stesso tempo sia pure in maniera confusa, spesso inconsapevole e, molte volte, contraddittoria - è comunque alla ricerca di una nuova autenticità in cui l'io nudo lasci

49 Xavier Zubiri, El problema teologal del hombre: cristianismo (Madrid: Alianza Editorial - Fundación Xavier Zubiri, 1997), 18.

${ }^{50}$ Ibidem, 19. 
addietro la sua aggressività e le sue paure in una nuova unità con gli altri, con l'Universo, con l'UNO, con Dio.

Come non ritenere che possa essere proprio un rinnovato annuncio del Dio Uno e Trino, di Dio Amore - declinato esistenzialmente in rapporti nuovi e trinitari - ciò che l'uomo d'oggi attende nella sua problematica ricerca di pienezza? In quest'ottica, il carisma dell'unità - da più di settanta anni è uno strumento che veicola tale visione con tutte le sue conseguenze per la Chiesa e per l'umanità nelle sue molteplici dimensioni: come spiritualità, come prassi esistenziale, come progetto sociale e culturale perché in esso si possono cogliere quelle caratteristiche di un'antropologia trinitaria che pienamente incardinata nell' humus del Magistero della Chiesa e della riflessione teologica contemporanea - possono offrire un contributo specifico alle esigenze dell'oggi.

"Immersa nella vita trinitaria, Lubich è stata spinta a dar forma trinitaria ad ogni realtà", dice iconicamente Zanghís1. Ed è proprio da qui che nasce una sintesi particolare che valorizza pienamente l'Incarnazione, mettendo in luce la persona umana nella sua essenza pura, vale a dire, vista a partire dal disegno eterno del Padre, dall'Uni-Trinità. Si tratta di una prospettiva genuinamente biblica ed escatologica, in quanto svela il nostro essere nell'essere del Verbo incarnato-risorto, ciò che già siamo in Lui e ciò che saremo in eterno, quella persona pienamente dispiegata in amore ad immagine delle persone divine nell'Amore infinito della Trinità ${ }^{52}$.

L'evento della croce, con il lacerante grido d'abbandono del Figlio, è la porta d'accesso, la chiave di lettura - sempre misteriosa e sfidante per la ragione umana - di questo infinito mistero. E alcuni brani di un testo densissimo di Lubich, che riportiamo, possono permetterci l'accesso alla novità precipua del carisma dell'unità:

${ }^{51}$ Giuseppe M. Zanghí, Gesù abbandonato maestro di pensiero (Roma: Città Nuova, 2008), 59.

${ }^{52}$ Afferma Zanghí: "l'essere persona è un dono offerto all'uomo, dono che ha il suo inizio aurorale nella condizione d'individualità - la condizione edenica dell'Adamo-Eva, piagata e 'incurvata' poi dal peccato - per giungere, e solo nel Cristo, nel meriggio della persona compiuta che può chiamare Dio: Padre. Quella persona nella quale cielo e terra s'incontrano: l'eterna 'idea' che Dio ha di me e la 'realtà' di me creatura: pienamente uno perfettamente distinte. È la theanthropia partecipata alla creatura umana. È il dischiudersi del cammino di tutta la creazione verso una condizione conveniente al 'compimento' dell'uomo in Cristo" [Giuseppe Maria Zanghí, “Quale uomo per il terzo millennio?”, Nuova Umanità 134 (2001): 273]. 
Gesù, soprattutto nell'evento pasquale della passione che frutta la redenzione e l'effusione dello Spirito, ci rivela l'Essere della Trinità come Amore. Di questo mistero, infatti, egli crocifisso è l'immagine visibile, la traduzione perfetta nel mondo creato.

Gesù abbandonato è il miracolo dell'annullamento di ciò che è perché l'essere sia. Miracolo comprensibile solo da chi conosce l'Amore e sa che nell'Amore tutto e nulla coincidono. Per questo egli, potenza di Dio e Sapienza di Dio (1 Cor 1, 24), ci è apparso quale finestra di Dio spalancata sul mondo e finestra dell'umanità attraverso la quale si può contemplare Dio. [...]

Sono tre le Persone della Santissima Trinità, eppure sono Uno perché 1'Amore non è ed è nel medesimo tempo. Il Padre è distinto dal Figlio e dallo Spirito, pur contenendo in Sé Figlio e Spirito. Uguale quindi allo Spirito, che contiene in Sé e Padre e Figlio, e al Figlio che contiene in Sé Padre e Spirito Santo. Nella relazione delle Persone divine, cioè, ciascuna, perché Amore, compiutamente è non essendo: perché è tutta pericoreticamente nelle altre, in un eterno donarsi. [...]

Analoga realtà è stata impressa da Dio nel rapporto fra gli uomini, lo abbiamo avvertito da quando Dio ci ha donato la sua luce. Ho sentito io stessa, anni addietro, d'essere stata creata in dono a chi mi sta vicino e chi mi sta vicino creato da Dio in dono a me, come il Padre nella Trinità è tutto per il Figlio e il Figlio è tutto per il Padre. E per questo il rapporto tra noi è lo Spirito Santo, lo stesso rapporto che c'è fra le Persone della Trinità. È la vita della Santissima Trinità che dobbiamo cercare di imitare, amandoci tra noi, con l'amore effuso dallo Spirito nei nostri cuori, come il Padre e il Figlio si amano tra loro ${ }^{53}$.

Da questa chiave di lettura - che è insieme esperienza mistica e riflessione intellettuale - emergono alcuni capisaldi per un nuovo umanesimo e per una antropologia all'altezza di quelle sfide dei nostri tempi precedentemente sottolineate $^{54}$ :

${ }^{53}$ Chiara Lubich, "Spiritualità dell'unità e vita trinitaria. Lezione per la laurea honoris causa in teologia", Nuova Umanità 151 (2004): 14-15. Dottorato attribuitole dalla Università di Trnava, la cui cerimonia è avvenuta a Castelgandolfo, il 23 giugno 2003.

${ }^{54}$ Cf. Chiara Lubich, "Gesù Maestro”, Nuova Umanità 180 (2008): 607-612; Chiara Lubich, Una famiglia per rinnovare la società (Roma: Città Nuova, 1993), 109-120; Chiara Lubich, “All'UNESCO nel conferimento del premio per l'educazione alla pace”, Nuova Umanità 108 (1996): 639-644; Chiara Lubich, Una cultura nuova per una nuova società. Discorsi in occasione del conferimento di lauree h. c., congressi e convegni 1996-2001 (Roma: Città Nuova, 2002), 161-173; Chiara Lubich, Inaugurazione del $1^{\circ}$ corso dell'Istituto Superiore di Cultura (Montet, 15/8/2001; Rocca di Papa: Archivio Movimento dei Focolari); Centro Chiara 
- Una visione dell 'essere: l'essere come amore. Al di là dell'ontificazione dell'essere imposta dalla ragione - che finisce per catturarlo identificandolo con se stessa - e senza approdare all'essere-nulla heideggeriano che invece lo dissipa, l'essere come amore mostra il volto di un Dono infinito (il non dovuto dell' amore che è Dio stesso, come dice Benedetto $\mathrm{XVI}^{55}$ ) che, essendo, fa essere nella misura che è ricevuto come tale.

- Una visione del soggetto: il soggetto Gesù. I molti fatti uno in Gesù pur rimanendo distinti ("tutti voi infatti siete uno in Cristo Gesù": Gal 3, 28). L'Anima, lo chiama Chiara Lubich, “quell'Una che ci univa tutti" 56 , il Risorto in mezzo a noi, come realtà escatologica, nel quale l'amore reciproco ci inserisce.

- Una visione della relazionalità: i "rapporti trinitari". Jean-Luc Marion scriveva: "Io ricevo la mia individualità insostituibile dall'avanzare dell'altro nel suo sguardo; io mi ricevo, allora, come insostituibile dalla sua propria estasi" ${ }^{57}$. E Zubiri: "l'uomo è assoluto in alterità" ${ }^{58}$. Ma l'alterità - splendidamente scoperta e approfondita dal personalismo - solo sarà feconda se viene concepita come rapporto costitutivo e costituente e quindi se vissuta come comunione.

- Una visione del sapere: la sapienza come pensiero di Cristo ("noi abbiamo il pensiero di Cristo": 1 Cor 2, 16).

- Una visione della spiritualità: la spiritualità di comunione, basata nel trinomio: Dio-il fratello-io.

- Un progetto culturale e sociale: "Che tutti siano Uno".

Il tutto può essere sintetizzato con una immagine molto efficace di Chiara che può esprimere con chiarezza il dinamismo spirituale e antropologico di questa prospettiva: Il castello esteriore, dove è evidente il riferimento a Teresa d'Avila:

Ma è venuto il momento, almeno questa è la nostra vocazione, di scoprire, illuminare, edificare, oltre il castello interiore anche il castello esteriore. Noi

Lubich - Istituto Universitario Sophia, Dottorati honoris causa conferiti a Chiara Lubich (Roma: Città Nuova, 2016), 320-329.

55 Benedetto XVI, Caritas, n. 34.

${ }^{56}$ Chiara Lubich, "Paradiso '49”, Nuova Umanità 177 (2008): 288.

57 Jean Luc Marion, Prolegómenos a la caridad (Madrid: Caparros editores, 1993), 115.

58 Zubiri, Tres dimensiones, 69. 
vediamo tutto il Movimento come un castello esteriore, dove Cristo è presente e illumina ogni parte di esso dal centro alla periferia. E se pensiamo che questa nuova spiritualità che Dio dona oggi alla Chiesa arriva anche ai responsabili della società e della Chiesa, comprendiamo subito che questo carisma non fa solo dell'Opera nostra un castello esteriore, ma tende a farlo del corpo sociale ed ecclesiale ${ }^{59}$.

\section{Spunti conclusivi per una corrispondente prassi educativa}

Per concludere solo un breve accenno ad alcuni spunti che - a partire da quanto detto finora - se ne possono ricavare anche sul piano pedagogico. In molte occasioni Lubich si è trovata a sottolineare come il Movimento dei Focolari e la sua storia possono essere visti come un grande, straordinario evento educativo in cui sono presenti tutti i fattori dell'educazione ed è pure evidente la presenza di una teoria dell'educazione, di una ben delineata pedagogia che fonda il suo agire educativo.

In particolare - nella lezione tenuta, il 10 novembre 2000, presso la Catholic University of America di Washington, in occasione del conferimento del dottorato honoris causa in pedagogia - Lubich ripercorre "gli elementi caratteristici della nostra pedagogia legati ai cardini principali della spiritualità che viviamo"60.

$\mathrm{E}$ in una articolata esposizione, mostra le implicazioni educative che emergono da ogni aspetto della spiritualità dell'unità e dall'antropologia che ne deriva e che da tanti decenni sono oggetto di sperimentazione all'interno del Movimento - in ogni contesto di formazione - e al suo esterno in tutte quelle realtà educative che ad esso si ispirano - in tanti diversi contesti culturali - e che possiamo sintetizzare nei seguenti punti principali:

- La necessità che sia una vera intenzionalità a guidare l'educatore lungo un itinerario di formazione che, oramai, non può non essere che personale e comunitario al medesimo tempo.

59 Chiara Lubich, Una via nuova (Roma: Città Nuova, 2007), 28-29.

${ }^{60}$ Chiara Lubich, "Il carisma dell'unità e la pedagogia. Estratto della lezione svolta, il 10 novembre 2000, presso la Catholic University of America di Washington, in occasione del conferimento del dottorato honoris causa in pedagogia", in: Chiara Lubich, La Dottrina spirituale (Milano: Mondadori, 2001), 227-230. 
- La riscoperta della dimensione della paternità: una scoperta di portata enorme se si pensa che l'eclissi del Padre (l'Assoluto) che una certa cultura ha tentato di affermare sul piano teorico e pratico ha favorito anche un'eclissi del padre e cioè del principio di autorità spesso con conseguenze disastrose soprattutto per le nuove generazioni.

- Il bisogno di recuperare quella autorevolezza sul piano del rapporti umani ed educativi, necessaria per arginare il relativismo etico e l'assenza di regole sia nella vita individuale che nelle relazioni interpersonali e sociali sempre più fonte di problemi e violenze di ogni genere.

- Il riconoscimento di ogni persona nella sua identità unica, originale e irripetibile che va aiutata a maturare educandola alla responsabilità, all'impegno anche mediante richieste esigenti.

- Il riferimento alla regola aurea di Comenius "insegnare tutto a tutti", ma secondo le esigenze pedagogiche della gradualità che non anticipa i tempi e rispetta lo sviluppo di ciascuno.

- Il puntare decisamente a quell'unità esistenziale favorendo il superamento della frammentazione che l'uomo prova spesso nella sua relazione con se stesso, con l'altro, con la società, con Dio.

- Il favorire costantemente il passaggio dalla fase iniziale di dipendenza da una legge eteronoma a quella di interiorizzazione di tale legge superando le difficoltà e gli ostacoli da vedere come mezzi necessari di crescita e maturazione.

- La costante attenzione a non favorire quelle forme di iperprotettività che tendono a preservare soprattutto i minori da qualsiasi difficoltà, lasciandoli così impreparati di fronte alle inevitabili prove, errori e fallimenti della vita rendendoli passivi rispetto alle responsabilità che ognuno deve assumersi di fronte a se stesso, al prossimo, alla società.

- Il superamento delle anguste modalità esistenziali dell'Io autocentrato per puntare al continuo autotrascendimento, ad un oltrepassamento verso l'altro nella scoperta della reciprocità che arricchisce e fa liberi.

- Il mantenere sempre viva la finalità da sempre assegnata all'educazione che è il formare l'uomo e la sua autonomia ma nell'orizzonte della formazione dell'uomo-relazione in vista della costruzione della comunità in cui egli vive. 
In fondo si tratta di creare dappertutto luoghi e contesti in cui le modalità di relazione siano improntate all'amore che solo può far fiorire e maturare ciascuno secondo le caratteristiche che gli sono proprie e uniche. Significativo a questo proposito quanto ha scritto Lubich nel 2004:

L'educazione è fondamentale per guidare la persona verso il suo dover essere, un fine che si ritiene valido per l'uomo e per l'umanità. Ma cosa occorre per educare in questo modo? Come occorre la primavera perché un giardino fiorisca, allo stesso modo è necessario quel calore che nasce dall'amore per far germogliare le verità che sono insite nell'uomo ${ }^{61}$.

Il tutto in vista di quella grande utopia che è il sognare un mondo diverso, solidale, attento alla salvaguardia delle diversità ma che punti decisamente verso l'unità. In tale prospettiva, pertanto, non si può non riferirsi anche a Maria e al ruolo da lei svolto nel piano di Dio dell'incarnazione del Verbo. È lei - in quanto icona della Trinità e immagine sublime della natura umana pienamente personalizzata ("l'umanità realizzata" - dirà Lubich) - a rappresentare per gli uomini della seconda modernità, come in tutte le altre epoche, quel nuovo modello antropologico cui - anche se spesso inconsapevolmente - anelano.

\section{Bibliografia}

Bauman, Zygmunt. Individualmente insieme. Reggio Emilia: Diabasis, 2008. Benedetto XVI. Caritas in Veritate. Città del Vaticano: Libreria Editrice Vaticana, 2009.

Carella, Nino. Silvia prima di Chiara. La ricerca di una strada nuova. Roma: Città Nuova, 2014.

Centro Chiara Lubich - Istituto Universitario Sophia. Dottorati honoris causa conferiti a Chiara Lubich. Roma: Città Nuova, 2016.

Chatel, Francesco. "Alla scuola di chi?". Nuova Umanità 180 (2008): 613-621.

Coda, Piero. "I movimenti ecclesiali, dono dello Spirito". In: Pontificium Consilium pro Laicis, I movimenti nella Chiesa, 77-103. Città del Vaticano: Libreria Editrice Vaticana, 1999.

${ }^{61}$ Chiara Lubich, Messaggio al Convegno di SportMeet, Vienna (Austria), settembre 2004. Rocca di Papa: Archivio Movimento dei Focolari. 
Coda, Piero. La Chiesa è il Vangelo. Alle sorgenti della teologia di papa Francesco. Città del Vaticano: Libreria Editrice Vaticana, 2017.

Concilio Vaticano II. Gaudium et spes. Città del Vaticano: Libreria Editrice Vaticana, 1965.

Congregazione per la dottrina della fede. Iuvenescit Ecclesia. Città del Vaticano, 16 maggio 2016.

Falmi, Donato. "Presentazione della collana Studi e Documenti". In: Qui c'è il dito di Dio. Carlo de Ferrari e Chiara Lubich: il discernimento di un carisma, ed. Lucia Abignente, 5-8. Roma: Centro Chiara Lubich - Città Nuova, 2017.

Fariñas Dulce, Maria José. "Neoindividualismo y desigualdad". El País, mercoledì, 5 gennaio 2005. https://elpais.com/diario/2005/01/05/opinion/1104879608 850215.html [20.09.2018].

Francesco. Evangelii gaudium. Città del Vaticano: Libreria Editrice Vaticana, 2013.

Francesco. Laudato sii. Città del Vaticano: Libreria Editrice Vaticana, 2015.

Francesco. Lumen Fidei. Città del Vaticano: Libreria Editrice Vaticana, 2013.

Francesco. Veritatis gaudium. Città del Vaticano: Libreria Editrice Vaticana, 2018.

Giovanni Paolo II. "V Simposio dei Vescovi d'Europa". L'Osservatore Romano, 7 ottobre 1982.

Giovanni Paolo II. Novo Millennio Ineunte. Città del Vaticano: Libreria Editrice Vaticana, 2001.

Giovanni Paolo II. Redemptor hominis. Città del Vaticano: Libreria Editrice Vaticana, 1979.

Hünermann, Peter. Uomini secondo Cristo oggi. L'antropologia di papa Francesco. Città del Vaticano: Libreria Editrice Vaticana, 2017.

Lubich, Chiara. "Spiritualità dell'unità e vita trinitaria. Lezione per la laurea honoris causa in teologia". Nuova Umanità 151 (2004): 11-20.

Lubich, Chiara. "All'UNESCO nel conferimento del premio per l'educazione alla pace". Nuova Umanità 108 (1996): 639-644.

Lubich, Chiara. "Gesù Maestro". Nuova Umanità 180 (2008): 607-612.

Lubich, Chiara. "Il carisma dell'unità e la pedagogia. Estratto della lezione svolta, il 10 novembre 2000, presso la Catholic University of America di Washington, in occasione del conferimento del dottorato honoris causa in pedagogia". In: Chiara Lubich, La Dottrina spirituale, 227-230. Milano: Mondadori, 2001.

Lubich, Chiara. "Paradiso '49". Nuova Umanità 177 (2008): 285-296.

Lubich, Chiara. Educazione come vita, ed. A. Vincenzo Zani. Brescia: La Scuola, 2010.

Lubich, Chiara. Inaugurazione del $1^{\circ}$ corso dell'Istituto Superiore di Cultura. Montet, 15/8/2001. Rocca di Papa: Archivio Movimento dei Focolari. 
Lubich, Chiara. Messaggio al Convegno di SportMeet, Vienna (Austria), settembre 2004. Rocca di Papa: Archivio Movimento dei Focolari.

Lubich, Chiara. Una cultura nuova per una nuova società. Discorsi in occasione del conferimento di lauree h. c., congressi e convegni 1996-2001. Roma: Città Nuova, 2002.

Lubich, Chiara. Una famiglia per rinnovare la società. Roma: Città Nuova, 1993.

Lubich, Chiara. Una via nuova. Roma: Città Nuova, 2007.

Marion, Jean-Luc. Prolegómenos a la caridad. Madrid: Caparros editores, 1993.

Nussbaum, Martha C. Non per profitto. Bologna: Il Mulino, 2011.

Paolo VI. Populorum progressio. Città del Vaticano: Libreria Editrice Vaticana, 1967.

Quartana, Pino. "Introduzione. Avere il Maestro tra di noi”. In: Chiara Lubich, Una famiglia per rinnovare la società, 105-108. Roma: Città Nuova, 1993.

Rifkin, Jeremy. La civiltà dell'empatia. Milano: Mondadori, 2010.

Steiner, George. La lezione dei maestri. Milano: Garzanti, 2004.

Urs von Balthasar, Hans. Teologica. Vol. III. Milano: Jaca Book, 1992.

Zanghí, Giuseppe Maria. "Il pensare come amore. Verso un nuovo paradigma culturale". Nuova Umanità 145 (2003): 1-19.

Zanghí, Giuseppe Maria. "Quale uomo per il terzo millennio?". Nuova Umanità 134 (2001): 247-277.

Zanghí, Giuseppe Maria. Gesù abbandonato maestro di pensiero. Roma: Città Nuova, 2008.

Zanghí, Giuseppe Maria. Leggendo un carisma. Chiara Lubich e la cultura. Roma: Città Nuova, 2015.

Zanghí, Giuseppe Maria. Notte della cultura europea. Roma: Città Nuova, 2007.

Zubiri, Xavier. El problema teologal del hombre: cristianismo. Madrid: Alianza Editorial - Fundación Xavier Zubiri, 1997.

Zubiri, Xavier. Tres dimensiones del ser humano: individual, social, histórica, ed. Jordi Corominas. Madrid: Alianza Editorial - Fundación Xavier Zubiri, 2006. 